\title{
Efeitos de exercícios do método Pilates na força muscular respiratória de idosas: um ensaio clínico
}

\author{
Effects of the Pilates method exercises in respiratory muscle strength of elderly women: \\ a clinical trial
}

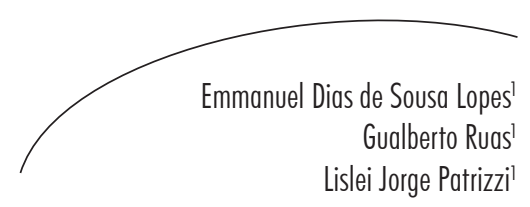

\section{Resumo}

Introducão: Dentre os sistemas do organismo, acredita-se que o respiratório seja o que envelhece mais rapidamente, devido à maior exposição a poluentes ambientais ao longo dos anos. O método Pilates surge como forma de condicionamento físico particularmente interessado em proporcionar bem-estar geral ao indivíduo. Objetivo: Avaliar os efeitos de exercícios do método Pilates na força muscular respiratória de idosas antes e após 11 semanas de treinamento. Métodos: Trata-se de ensaio clínico, longitudinal e prospectivo. Foram selecionadas sete mulheres com idade igual e superior a 60 anos e com autonomia cognitiva preservada. A Prova de Função Pulmonar (Espirometria) foi realizada por meio do espirômetro marca Vitalograph ${ }^{\circledR}$ modelo 8600. A força muscular respiratória foi obtida pelas técnicas de medidas da pressão inspiratória máxima e pressão expiratória máxima, por meio de um manovacuômetro analógico da marca Gerar. O período experimental foi de 11 semanas. Para a análise comparativa dos dados obtidos nas avaliações, foi aplicado o teste $t$ pareado e nível de significância de 5\%. Resultados: Os resultados do estudo mostraram aumento significativo $(\mathrm{p} \leq 0,01)$ em relação à pressão expiratória máxima de $46 \pm 18$ para $75 \pm 29 \mathrm{cmH} 2 \mathrm{O}$. Conclusão: Os resultados apresentaram aumento significativo na pressão expiratória máxima, sendo o método Pilates uma das práticas recomendadas à população idosa.

\section{Registro Ensaio Clínico: ClinicalTrials.gov ID: NCT02189044}

\section{Abstract}

Introduction: Among the systems of the organism, it is believed that the respiratory system is the one that gets older faster due to the greater exposure to environmental pollutants over the years. The Pilates method has emerged as a form of fitness particularly providing general welfare to the individual. Objective: To evaluate the effects of Pilates' exercises on respiratory muscle strength in elderly women before and after eleven weeks of training. Methods: This is a clinical, longitudinal, prospective trial study. We have studied seven women aged 60 and over, with preserved autonomy

Palavras-chave: Idoso. Exercícios Respiratórios. Força Muscular.

\footnotetext{
Curso de Fisioterapia. Universidade Federal do Triângulo Mineiro. Uberaba, MG, Brasil.
} 
and cognitive skills. The Pulmonary Function Test (Spirometry) was performed using the Vitalograph ${ }^{\circledR}$ spirometer, model 8600. Respiratory muscle strength was obtained by technical measurements of maximal inspiratory pressure and maximal expiratory pressure, through analog Gerar manometer. The experiment lasted eleven weeks. For comparative analysis of data obtained in the evaluations, we applied the paired $t$ test and a significance level of $5 \%$. Results: The results of this study showed significant increase $(\mathrm{p} \leq 0.01)$ compared to maximal expiratory pressure of $46 \pm 18$ for $75 \pm 29 \mathrm{cmH} 2 \mathrm{O}$. Conclusion: The results showed significant increase in maximal expiratory pressure, so the Pilates method is one of the practices recommended for the elderly population.
Key words: Elderly. Breathing Exercises. Muscle Strength.

Registro Ensaio Clínico: ClinicalTrials.gov ID: NCT02189044

\section{INTRODUÇÃO}

Dentre os sistemas do organismo, acreditase que o respiratório seja o que envelhece mais rapidamente, devido à maior exposição a poluentes ambientais ao longo dos anos. ${ }^{1} \mathrm{De}$ acordo com Kim \& Sapienza, ${ }^{2}$ uma das principais mudanças no sistema respiratório com o avançar da idade é a diminuição do recolhimento elástico dos pulmões e da complacência da caixa torácica. Essas alterações estão relacionadas às mudanças na quantidade e na composição dos componentes dos tecidos conjuntivos do pulmão, como elastina, colágeno e proteoglicanos.

Estudos demonstram que a idade é um preditor negativo das forças musculares respiratórias tanto em homens quanto em mulheres. ${ }^{3,4}$ Observamse declínio da capacidade vital forçada, volume expiratório forçado no primeiro segundo e fluxo expiratório forçado, bem como aumento na capacidade residual funcional e volume de reserva expiratório, que se relacionam com a redução no recolhimento elástico pulmonar e a diminuição da complacência da caixa torácica. ${ }^{5}$

Essas alterações são responsáveis pela redução da força muscular respiratória, prejudicando não só a função de bomba ventilatória como também as funções não ventilatórias do sistema respiratório. As funções não ventilatórias incluem: tossir, espirrar, falar, cantar, realizar a manobra de Valsalva e outras funções que são acompanhadas pelo esforço expiratório. A redução da força muscular respiratória pode ser amenizada com a prática de exercícios físicos. ${ }^{2}$
Baseando-se em princípios da cultura oriental - como ioga, artes marciais e meditação -, o Pilates se caracteriza pela tentativa do controle dos músculos envolvidos nos movimentos da forma mais consciente possível. ${ }^{6}$ Segundo McNeill, 7 o Pilates, técnica criada no início da década de 1920 por Joseph Pilates, se baseia numa integração geral de todos os movimentos do corpo, envolvendo contrações musculares de alto ou baixo limiar, e explora exercícios de amplitude de movimento completa ou com a articulação em posição neutra. $O$ método se baseia no conceito da contrologia, em que todos os movimentos do corpo devem ser totalmente conscientes, entendendo seus princípios de equilíbrio e gravidade nos diferentes momentos. ${ }^{8}$

O método Pilates surge como forma de condicionamento físico particularmente interessado em proporcionar bem-estar geral ao indivíduo, sendo capaz de proporcionar força, flexibilidade, boa postura, controle postural, consciência corporal e percepção do movimento.

Os exercícios que compõem o método envolvem contrações isotônicas (concêntricas e excêntricas) e sobretudo isométricas, com ênfase no que Joseph Pilates denominou powerbouse (ou centro de força). Este centro de força é composto pelos músculos abdominais, glúteos e paravertebrais lombares, que são responsáveis pela estabilização estática e dinâmica do corpo. Então, durante os exercícios, a expiração é associada à contração do diafragma, do transverso abdominal, dos multífidos e dos músculos do assoalho pélvico. ${ }^{10,11}$ 
Os benefícios dos exercícios do método Pilates e seu possível impacto nas alterações da força muscular respiratória em idosos não são encontrados na literatura. Justifica-se, portanto, a realização deste estudo, que teve como objetivo avaliar os efeitos de exercícios do método Pilates na força muscular respiratória de idosas antes e após 11 semanas de treinamento.

\section{METODOLOGIA}

Este estudo é um ensaio clínico, longitudinal e prospectivo realizado no Laboratório de Cinesioterapia e Cinesiologia do Curso de Fisioterapia da Universidade Federal do Triângulo Mineiro (UFTM). Todas as participantes assinaram o Termo de Consentimento Livre e Esclarecido. A pesquisa teve início após aprovação do Comitê de Ética em Pesquisa da UFTM, protocolo no 2.147/ 2012.

\section{Sujeitos}

Fez-se inicialmente o convite a todos os alunos (quatro homens e 20 mulheres, totalizando 24 alunos na Turma I) matriculados no Programa Universidade Aberta a Terceira Idade (UATI/ UFTM), no período de agosto a outubro de 2012.

A seleção baseou-se nos seguintes critérios de inclusão: (1) idade igual e superior a 60 anos; (2) não praticante de atividade física regular; (3) disponibilidade para frequentar as aulas de Pilates duas vezes por semana (além do horário UATI); e (4) autonomia cognitiva preservada (critério de inclusão para o programa UATI-UFTM). E também nos critérios de exclusão: (1) histórico de doenças que afetassem diretamente a postura (hérnia discal e deformidades angulares da coluna); (2) doenças cardiovasculares, respiratórias e neurológicas que impedissem a realização dos exercícios de Pilates; e (3) duas faltas consecutivas ou um total de três faltas durante o tempo proposto para o treinamento estabelecido.

Sendo assim, a metodologia proposta para este estudo foi aplicada em sete mulheres com idade igual e superior a 60 anos.

\section{Avaliação/procedimentos}

Os instrumentos de avaliação utilizados foram: (1) ficha de avaliação inicial, (2) espirômetro e (3) manovacuômetro.

A ficha de avaliação inicial e a espirometria foram aplicadas para a seleção das participantes. Com base na ficha de avaliação inicial, foi possível avaliar os aspectos relacionados à condição de saúde das participantes (doenças associadas, pressão arterial sistêmica, frequência respiratória e cardíaca, avaliação postural), bem como obter informações sobre os antecedentes pessoais e familiares e medicamentos em uso.

A prova de função pulmonar (espirometria) foi realizada por meio do espirômetro marca Vitalograph ${ }^{\circledR}$ modelo 8600. O equipamento foi calibrado e os testes foram realizados por apenas um examinador treinado e habilitado, com instruções e comandos padronizados de voz, conforme normas preconizadas pelo I Consenso Brasileiro sobre Espirometria. ${ }^{12}$

A espirometria foi realizada para descartar qualquer distúrbio ventilatório que afetasse na realização dos exercícios propostos.

A mensuração da força muscular respiratória ocorreu por meio de um manovacuômetro analógico (Ger-Ar ${ }^{\circledR}$, São Paulo, Brasil), com escalas de -300 a $+300 \mathrm{cmH}_{2} \mathrm{O}$ (devidamente calibrado), equipado com traqueia de plástico de $16 \mathrm{~cm}$ de comprimento e $2,4 \mathrm{~cm}$ de diâmetro interno, bocal de plástico rígido e orifício de fuga de aproximadamente $2 \mathrm{~mm}$ de diâmetro para dissipação das pressões geradas pela musculatura da face e da orofaringe. ${ }^{13}$ Foram obtidas as medidas de pressão inspiratória máxima (PImáx) e pressão expiratória máxima (PEmáx), realizadas no máximo cinco vezes, aceitáveis e reprodutíveis, sendo na posição ortostática, com uso de clipe nasal, por um tempo de sustentação de três segundos.

O valor da PImáx foi obtido a partir do volume residual, e a PEmáx, a partir da capacidade pulmonar total. As voluntárias receberam o devido incentivo verbal e, caso 
houvesse diferença maior que 10\% entre uma medida e outra, nova manobra era realizada, considerando-se o maior valor obtido desde que esse não fosse o último. ${ }^{14}$

Intervenção terapêutica - método Pilates

O treinamento por meio de exercícios do método Pilates foi realizado em 11 semanas. As participantes foram orientadas a não faltar e a participar das aulas duas vezes por semana. Cada aula foi composta por cinco exercícios com duração de 40 minutos cada aula.

Durante as quatro primeiras semanas $\left(1^{\mathrm{a}}\right.$ a $4^{\mathrm{a}}$ semanas), os exercícios realizados foram: gato, mesinha, single leg circle, movimentos pélvicos, contração dos glúteos e para encerramento foi realizado alongamento de cadeia posterior com faixa.

Nas quatro semanas seguintes $\left(\begin{array}{lll}5^{a} & \text { a } & 8^{a}\end{array}\right.$ semanas), iniciou-se uma nova série de exercícios, sendo eles: ponte, mesinha, ponte hip roll com leg extension, abdominal - balf hollup, oblíquos e, ao final, alongamento - série de Willian's.

Nas últimas três semanas do treinamento $\left(9^{\mathrm{a}}\right.$ a $11^{a}$ semanas), foi proposta nova série de exercícios, que envolveu: beaststroke, apoio calcanhar, ponte - bip roll com leg extension, bundred e agachamento com bastão na parede. Todos os exercícios foram realizados durante a expiração.

\section{Análise estatística}

Realizou-se análise descritiva dos dados obtidos, apresentando média e desvio-padrão. Para a análise comparativa dos dados obtidos nas avaliações pré e pós-intervenção, foi aplicado o teste $t$ pareado (após confirmada a normalidade na distribuição da amostra - teste Shapiro Wilk). O nível de significância adotado foi de $5 \%$. O programa estatístico utilizado foi o SPSS 18.0.

\section{RESULTADOS}

Na tabela 1 são apresentadas a idade e medidas antropométricas das voluntárias praticantes do método Pilates.

A tabela 2 apresenta os valores de PImáx obtido e previsto, PEmáx obtido e previsto e os valores de $\mathrm{p}$ da amostra $(\mathrm{n}=7)$ antes e após 11 semanas de treinamento.

Tabela 1. Caracterização da amostra em relação ao número de participantes, sexo e as médias de idade, peso, estatura e IMC. Uberaba-MG, 2012.

\begin{tabular}{lc}
\hline \multicolumn{1}{c}{ Variável } & Amostra \\
\hline Número de participantes (n) & 7 \\
Sexo feminino (n) & 7 \\
Idade (anos) média + dp & $64 \pm 6$ \\
Peso $(\mathrm{kg})$ média + dp & $71 \pm 8$ \\
Estatura $(\mathrm{cm})$ média + dp & $154 \pm 6$ \\
IMC $\left(\mathrm{kg} / \mathrm{m}^{2}\right)$ média $+\mathrm{dp}$ & $29 \pm 4$ \\
\hline
\end{tabular}

$\mathrm{IMC}=$ índice de massa corporal. 
Tabela 2. Força muscular respiratória $(\mathrm{n}=7)$ determinada por meio de medidas da pressão inspiratória máxima (PImáx) e pressão expiratória máxima (PEmáx). Uberaba-MG, 2012.

\begin{tabular}{lccc}
\hline & Pré & Pós & p-valor \\
PImáx obtido $(\mathrm{cmH} 2 \mathrm{O})$ & $73( \pm 31)$ & $116( \pm 65)$ & 0,06 \\
PImáx predito $(\mathrm{cmH} 2 \mathrm{O})$ & $79( \pm 2)$ & $79( \pm 2)$ & 0,5 \\
PEmáx obtido $(\mathrm{cmH} 2 \mathrm{O})$ & $46( \pm 18)$ & $75( \pm 29)$ & 0,0001 \\
PEmáx predito $(\mathrm{cmH} 2 \mathrm{O})$ & $78( \pm 3)$ & $78( \pm 3)$ & 0,5 \\
\hline
\end{tabular}

\section{DISCUSSÃO}

Com o avanço da idade, reduções ocorrem nos valores da PImáx e da PEmáx, e estas podem estar relacionadas com as alterações fisiológicas próprias do processo de envelhecimento, como mudanças na composição do tecido pulmonar e da caixa torácica, que acarretam diminuição da massa e da eficiência da musculatura respiratória. ${ }^{15}$

As mudanças que ocorrem a este nível são clinicamente relevantes porque a deterioração da função pulmonar está associada ao aumento da taxa de mortalidade. Além disso, o conhecimento sobre as mesmas contribui para a detecção e prevenção de disfunções respiratórias em idosos. ${ }^{6}$

Todas essas alterações estruturais que ocorrem com o processo de envelhecimento modificam a curvatura do músculo diafragma, provocando um efeito negativo em sua capacidade de gerar força. Isso é o que sugerem Polkey et al., ${ }^{16}$ na tentativa de justificar a diminuição significativa da força diafragmática de sujeitos idosos (67-81 anos), quando comparados a um grupo controle de jovens (21-40 anos).

Segundo Neder et al., ${ }^{3}$ os idosos têm regressão na massa muscular do diafragma e da musculatura acessória da respiração, e também apresentam menor resposta desses músculos a um mesmo nível de estimulação neural.

Durante a expiração em repouso, o recolhimento elástico passivo dos pulmões é o responsável por gerar a expiração. Entretanto, os músculos expiratórios são contraídos quando há necessidade de produzir pressão pulmonar durante atividades não-ventilatórias. ${ }^{17}$

Os resultados do presente estudo mostraram aumento significativo $(\mathrm{p} \leq 0,01)$ em relação à PEmáx, o que nos leva a considerar a validade da prática do método Pilates. Watsford et al., ${ }^{18}$ em trabalho realizado com indivíduos idosos, relataram aumento significativo da PEmáx, com diminuição da PImáx, após treinamento com caminhada.

A prática de exercícios físicos tem se mostrado eficiente na melhoria da musculatura respiratória. ${ }^{2}$

Estudo de Gonçalves et al. ${ }^{19}$ observou que exercícios físicos gerais apresentaram aumento significativo na força muscular respiratória $(p=0,0001)$. Rendas et al. ${ }^{20}$ avaliaram os efeitos de um programa de exercícios físicos gerais não orientados especificamente na musculatura respiratória e função pulmonar, e verificaram diferenças estatisticamente significativas $(\mathrm{p}=0,028)$ nos valores de PEmáx, corroborando o presente estudo, porém com o método Pilates.

Não foram encontrados na literatura trabalhos anteriores que relacionam a prática do método Pilates com a força da musculatura respiratória, mas estudos sobre a eficácia da prática de exercícios afirmam que, apesar das 
reduções de força esperadas com o processo de envelhecimento, quando dado o estímulo correto, a força muscular periférica pode ser melhorada em qualquer idade. ${ }^{21,22}$

Baseando-se em princípios da cultura oriental, como ioga, artes marciais e meditação, o Pilates se caracteriza pela tentativa do controle dos músculos envolvidos nos movimentos da forma mais consciente possível. Oito são os princípios básicos pelos quais o Pilates é executado, a saber: concentração, controle, centragem, respiração diafragmática, leveza, precisão, força e relaxamento, sendo que os exercícios são adaptados às condições do paciente e o aumento da dificuldade respeita as características e habilidades individuais. ${ }^{10,23,24}$

Em relação ao princípio que envolve a respiração, Joseph Pilates afirmava que frequentemente respiramos errado por utilizarmos apenas uma fração da capacidade do pulmão. Por isso, enfatizava em seu trabalho a respiração como o fator primordial no início do movimento, fornecendo a organização do tronco pelo recrutamento dos músculos estabilizadores profundos da coluna na sustentação pélvica e favorecendo o relaxamento dos músculos inspiratórios e cervicais. . $^{23,25,26}$

Embora os resultados da prática do método Pilates duas vezes por semana durante 11 semanas tenham mostrado melhores níveis de PEmáx entre mulheres com idade acima de 60 anos, o número de participantes do estudo não é significativo para afirmar que os exercícios baseados no método Pilates são indicados para os ganhos obtidos. É válido ressaltar que a exigência da frequência semanal (duas vezes na semana, além do dia direcionado para as atividades da UATI) dificultou a adesão dos alunos à pesquisa, em função das várias atividades já assumidas por eles, mesmo os autores disponibilizando três opções de horários. Pelo mesmo motivo, justificase a ausência do grupo controle. O tempo de intervenção foi estabelecido respeitando-se o calendário acadêmico; sendo assim, em função do período de férias, foi necessário finalizar a intervenção na $11^{a}$ semana.

Assim, sugere-se que novos estudos sejam realizados, contemplando amostras mais amplas, grupo controle, com variação no método e tempo de intervenção, incluindo, se possível, idosos do sexo masculino. ${ }^{27,28}$

\section{CONCLUSÃO}

Conclui-se que os exercícios do método Pilates proporcionaram aumento significativo da pressão expiratória máxima na população idosa estudada. Assim, o método Pilates é uma das práticas recomendadas para essa população.

\section{REFERÊNCIAS}

1. Belini MAV. Força muscular respiratória em idosos submetidos a um protocolo de cinesioterapia respiratória em imersão e em terra [trabalho de conclusão de curso]. Cascavél: Universidade Estadual do Oeste do Paraná, Centro de Ciências Biológicas e da Saúde; 2004.

2. Kim J, Sapienza CM. Implications of expiratory muscle strength training for rehabilitation of the elderly: tutorial. J Rehabil Res Dev 2005;42(2):211-24.

3. Neder JA, Andreoni S, Lerario MC, Nery LE. Reference values for lung function tests. II. Maximal respiratory pressures and voluntary ventilation. Braz Med Biol Res 1999;32(6):719-27.
4. Black LF, Hyatt RE. Maximal respiratory pressures: normal values and relationship to age and sex. Am Rev Respir Dis 1969;99(5):696-702.

5. Ruivo S, Viana P, Martins C, Baeta C. Efeito do envelhecimento cronológico na função pulmonar: comparação da função respiratória entre adultos e idosos saudáveis. Rev Port Pneumol [Internet]. 2009 [citado 07 Abr 2013];15(4):629-53. Disponível em: http://www.scielo.oces.mctes.pt/scielo.php?pid=S0873$21592009000400005 \&$ script=sci_arttext

6. Silva YO, Melo MO, Gomes LE, Bonezi A, Loss JF. Análise da resistência externa e da atividade eletromiográfica do movimento de extensão de 
quadril realizado segundo o método Pilates. Rev Bras Fisioter 2009;13(1):82-8.

7. McNeill W. Decision making in Pilates. J Bodyw Mov Ther 2011;15(1):103-07.

8. Kolynia IEGG, Cavalcanti SMB, Aoki MS. Avaliação isocinética da musculatura envolvida na flexão e extensão do tronco: efeito do método Pilates.Rev Bras Med Esporte 2004;10(6):487-90.

9. Blum, Charles L. Chiropractic and pilates therapy for the treatment of adultscoliosis. J Manip Physiol Ther 2002;25(4):E1-8.

10. Pires DC, De SÁ CKC. Pilates: notas sobre aspectos históricos, princípios, técnicas e aplicações. Efdeportes.com 2005;10(91):1-4.

11. Hodges PW, Richardson CA. Contraction of the abdominal muscles associated with movement of the lower limb. Phys Ther 1997;77(2):132-42.

12. Lemble A, Algranti E, Jansen JM, Valença LM, Nery LE, Mallozi M, et al. Pereira CAC, coordenador. I consenso brasileiro sobre espirometria. J Pneumol 1996;22(3):105-64.

13. Supinski G. Determination and interpretation of inspiratory and expiratory pressure measurements. Clin Pulm Med 1999;6(2):118-25.

14. American Thoracic Society, European Respiratory Society. ATS/ERS statement on respiratory muscle testing. Am J Respir Crit Care Med 2002;166(4):518-624.

15. Chaunchaiyakul R, Groeller H, Clarke JR, Taylor NAS. The impact of aging and habitual physical activity on static respiratory work at rest and during exercise. Am J Physiol Lung Cell Mol Physiol 2004;287(6):1098-106.

16. Polkey MI, Green M, Moxham J. Measurement of respiratory muscle strength. Thorax 1995;50:1131-5.

17. Simões, Rodrigo Polaquini, Auad MA, Dionísio J, Mazzonetto M. Influência da idade e do sexo na força muscular respiratória. Fisioter Pesqu 2007;14(1):36-41.

18. Watsford M, Murphy A, Matthew J, Coutts AJ. The effect of habitual exercise on respiratory- muscle function in older adults. J Aging Phys Act 2005;13(1):34-44.

19. Gonçalves MP, Tomaz CAB, Cassiminho ALF, Dura MF. Avaliação da força muscular inspiratória e expiratória em idosas praticantes de atividade física e sedentárias. Rev Bras Ciênc Mov 2006;14(1):37-44.

20. Rendas AB, Gamboa T, Ramilo T, Botelho AS, Bárbara C, Mota-carmo M. Respiratory muscle function in physically active elderly women. Arch Gerontol Geriatr 1996;22(2):123-30.

21. Newton RU, Hakkinen K, Hakkinen A, McCormck M, Volek J, Kraemer WJ. Mixed-methods resistance training increases power and strength of young and older men. Med Sci Sports Exerc 2002;34(8):1367-75.

22. Watsford ML, Murphy AJ, Pine MJ. The effects of ageing on respiratory muscle function and performance in older adults. J Sci Med Sport 2007;10(1):36-44.

23. Craig C. Pilates com a bola. 2. ed. São Paulo: Phorte; 2004.

24. Lange C, Unnithan V, Larkam E, Latta P. Maximizing the benefits of Pilates-inspired exercise for learning functional motor skills. J bodyw Mov Ther 2000;4(2):99-108.

25. Daltro F, Fernandes F. Curso de Sistema de Abordagem Corporal Fundamentado na Técnica de Pilates. Curso de Capacitação Profissional. CorporeCentro de Desenvolvimento Físico. Salvador 4 (2004).

26. Gallagher SP, Kryzanowska R. O método de Pilates de Condicionamento Físico. São Paulo: The Pilates Studio do Brasil; 2000.

27. Schulz KF, Altman DG. Moher D. CONSORT 2010 Statement: updated guidelines for reporting parallel group randomised trials. BMC Medicine, v. 8, n. 1, p. 18, 2010 .

28. Campbell MK, Piaggio G, Elbourne DR, Altman DG. Consort 2010 statement: extension to cluster randomised trials. BMJ: British Medical Journal, v. 345, 2012. 